\title{
Innovación docente en la asignatura Gestión Integral para reforzar la competencia transversal Instrumental Específica
}

Andrés Boza, Raúl Oltra, Llanos Cuenca

${ }^{a}$ Universitat Politècnica de València. Dpto. Organización de Empresas. aboza@omp.upv.es; roltra@doe.upv.es; 1lcuenca@omp.upv.es

\begin{abstract}
The teaching innovation presented in this paper has as main objective that students are able to make use of the most appropriate software solutions for a given business context, knowing their uses and possibilities of integration to improve an organization. All this in the context of the transversal competence "Specific Instruments". The innovation is based on the design of a set of activities to achieve this goal: i) Laboratory practices. To have contact with real ERP systems; ii) Fieldwork. To approach a business reality and apply the knowledge acquired to prescribe improvement proposals.

The indicators that include the scoring rubric used for its evaluation are: I1: Identifies advanced tools and their usefulness; I2: Handles advanced tools; I3: Selects and combines the right tools to prescribe an improvement in a real business context.

For each indicator, the evidence to be gathered from laboratory practices and field practices has been established.

The proposed activities have allowed the students to manage tools both in the laboratory and in real contexts. Also note that the use of the rubric has facilitated the design and structuring of activities for teachers.
\end{abstract}

Keywords: Design of activities, Scoring Rubric, Cross-curricular competence, Specific Instrumental

\footnotetext{
Resumen

La innovación docente presentada tiene como objetivo principal que el alumno sea capaz de hacer uso de las soluciones software más adecuadas para un determinado contexto empresarial, conociendo sus utilidades y las posibilidades de integración para la mejora de una organización. Todo ello en el contexto de la competencia transversal "Instrumental Especifica". La innovación se basa en el diseño de un conjunto de actividades para alcanzar dicho objetivo: i)Prácticas de laboratorio. Para tener contacto con sistemas ERP reales; ii) Trabajo de campo. Para acercarse a una realidad empresarial y aplicar los conocimientos adquiridos para prescribir propuestas de mejora.
} 
Los indicadores que incluye la rúbrica utilizada para su evaluación son: I1: Identifica las herramientas avanzadas y su utilidad; I2: Maneja las herramientas avanzadas; I3: Selecciona y combina las herramientas adecuadas para prescribir una mejora en un contexto empresarial real.

Para cada indicador se han establecido las evidencias a recoger de las prácticas de laboratorio y prácticas de campo realizadas.

Las actividades planteadas han permitido el manejo de las herramientas por parte de los alumnos tanto en el laboratorio como en contextos reales. También destacar que el uso de la rúbrica ha facilitado el diseño y estructuración de las actividades al profesorado.

Palabras clave: Diseño de actividades, Rúbrica, Competencia Transversal, Instrumental Especifica

\section{Introducción}

Una competencia representa lo que los participantes en la formación deben ser capaces de hacer al final de dicha formación (Boza y Cuenca, 2014). Las competencias específicas pertenecen a un área específica del conocimiento (en un grado o máster) y tienen como objetivo lograr un perfil específico. Las competencias transversales (también denominadas competencias genéricas) son genéricas y transferibles en una amplia variedad de aspectos personales, sociales, académicos y profesionales. Por lo tanto, contribuyen a una parte fundamental del perfil educativo (Cuenca et al., 2016).

La competencia transversal "Instrumental Específica" hace referencia al uso de las herramientas y tecnologías necesarias para el ejercicio profesional asociado a cada titulación. El estudiante será capaz de identificar las herramientas más adecuadas en cada caso, conociendo sus utilidades y siendo capaz de integrarlas y combinarlas para poder resolver un problema, realizar un proyecto o un experimento. Para el desarrollo de esta competencia podemos realizar algunas de las siguientes actividades formativas (UPV,2017): Prácticas de laboratorio, Problemas, Proyectos y Portafolio.

La inclusión de elementos que permitan reforzar esta competencia de una manera explicita mediante el diseño de nuevas actividades o el rediseño de las existentes es una labor compleja, así como, su posterior proceso de evaluación.

Para facilitar y unificar criterios para la evaluación de las competencias, la UPV ha creado tres rubricas con diferente nivel de dominio, primer nivel para $1^{\circ}$ y $2^{\circ}$ de grado, segundo nivel para $3^{\circ}$ y $4^{\circ}$ de grado y tercer nivel para master (ICE, 2015).

\subsection{Contexto académico}

La asignatura Gestión Integral se imparte en el Master de Ingeniería Informática de la Escuela Técnica Superior de Ingeniería Informática. Se trata de una asignatura incluida en el Módulo de Asignaturas Optativas de $2^{\circ}$ curso de master y el número de alumnos participantes en las actividades fueron 22 (9 curso 2016-2017 y 13 curso 2015-2016).

(c)) EY-NC-ND 2017, Universitat Politècnica de València 
La asignatura pone el foco en la gestión integrada de la información en las organizaciones que esta permitiendo una mejora significativa en sus procesos de negocio. En concreto se analizan y trabajan de forma principal los sistemas ERP (Enterprise Resource Planning) que participan en estos cambios y los profesionales informáticos en estos ámbitos se sitúan como actores principales en estos procesos de cambio empresarial.

Los sistemas ERP son sistemas de información diseñados para integrar y optimizar los principales procesos de negocio de la empresa, siendo aceptados por la industria como una solución práctica para lograr sistemas integrados de información (Oltra, 2012). Integran todas las áreas funcionales de la empresa, procesos y datos, en una única base de datos, incluyendo la definición de vistas y roles de usuarios. Los sistemas ERP han evolucionado para convertirse en sistemas más amplios y más inclusivos. Su desarrollo continuo ha dado lugar a varios tipos de sistemas ERP con un enfoque en la industria o sector específico (Boza et al., 2015).

En el contexto de la asignatura se han llevado a cabo diversas innovaciones (Boza y Cuenca, 2014).

Entre los contenidos de la materia se encuentran:

Los sistemas de gestión empresarial, su evolución hacia sistemas ERP, los procesos de negocio soportados por los sistemas ERP (compras, ventas, producción, recursos humanos...), el proceso de selección de un ERP, el proyecto de implantación, la gestión del cambio, el mercado de soluciones ERP, nuevas tendencias en el campo de los sistemas de gestión integral y casos prácticos.

\section{Objetivo}

El objetivo último es que los alumnos de la asignatura Gestión Integral trabajen la competencia transversal Instrumental Específica y sean evaluados de acuerdo a dicho trabajo.

Para ello, se ha diseñado un conjunto de actividades que incluyen prácticas de laboratorio y trabajo de campo con el propósito de que el alumno, en el contexto de la competencia, sea capaz de hacer uso de las soluciones software más adecuadas para un determinado contexto empresarial, conocer sus utilidades y posibilidades de integración para la mejora de una organización.

\section{Desarrollo de la innovación}

Como se indicó anteriormente, las actividades a desarrollar incluyen prácticas de laboratorio y trabajo de campo.

El objetivo de las prácticas de laboratorio consiste en que los alumnos tengan contacto con sistemas ERP reales, y que sean capaces de identificar las diferentes funciones que se pueden llevar a cabo en los diferentes módulos, las posibilidades de adaptación a las diferentes necesidades de las empresas mediante parametrización, el alcance de cada uno de ellos y las posibilidades de integración con otras herramientas. En concreto trabajan con el ERP Openbravo, y con el ERP SAP . 
En ambos sistemas, las prácticas se centran en que los alumnos realicen procesos sobre la aplicación ERP en concreto (Openbravo o SAP según el caso) al igual que los realizarían en una empresa real. De este modo, incluyen artículos, que luego deben fabricar, vender o comprar, según la práctica se centre en uno u otro módulo del sistema ERP. Además, ven las diferentes de posibilidades que ofrece un ERP a través de su modularidad, parametrización y configuración (Gil et al, 2010), para que pueda adaptarse a las necesidades particulares de cada organización.

Por otro lado, el objetivo del trabajo de campo es doble, en primer lugar, consiste en acercarse a una realidad empresarial visitando una mediana empresa, identificando sus principales procesos y realizando un análisis de la situación de su sistema de información. Además, el trabjo de campo tiene tambien como objetivo aplicar los conocimientos adquiridos para prescribir propuestas de mejora.

\subsection{Prácticas de laboratorio}

En prácticas los alumnos trabajan en el laboratorio con dos herramientas ERP que facilitan la gestión de la información en las organizaciones pero que tienen un carácter diferenciado respecto a elementos como el número de funcionalidades que proporciona cada una de ellas, la cobertura internacional, el precio de la solución ERP o la facilidad de uso. A su vez, cada herramienta incluye un conjunto de módulos que pueden o no ser implementados en las empresas dependiendo de las características y necesidades de la misma.

La primera de las soluciones puestas a disposición de los alumnos es el ERP Openbravo. Se trata de una solución instalada en un servidor del Departamento de Organización de Empresas a disposición de los alumnos durante las sesiones de prácticas y con el que pueden seguir trabajando de forma remota fuera de horas de laboratorio. Además, esta herramienta puede ser instalada en los equipos de los propios alumnos al tratarse de una solución de software libre disponible en http://wiki.openbravo.com/wiki/Main_Page. Los módulos principales son los de Configuración General, Gestión de Datos Maestros, Gestión de Compras, Gestión de Almacén, Gestión de Producción, Gestión del MRP, Gestión de Ventas, Gestión de Proyectos y Servicios y Gestión Financiera.

La segunda solución software ERP con la que trabajan los alumnos en el laboratorio es SAP $\mathrm{R} / 3$. Se trata de una herramienta instalada en servidores de SAP en Alemania y que están a disposición de la comunidad universitaria gracias a la participación de la UPV en SAP University Alliance. Esta alianza permite la utilización de herramientas de SAP así como facilita abundante información sobre soluciones y herramientas avanzadas (SAP UA 2017). $\mathrm{Al}$ igual que en el anterior caso, los alumnos tienen acceso al software en el laboratorio, así como de forma remota. Los módulos principales que se encuentran en SAP R/3 son Fianzas, Costos y Control, Ventas y Distribución, Gestión de Materiales, Producción y Recursos Humanos (Akhtar 2013).

En cada una de las prácticas de laboratorio el alumno trabaja con diferentes módulos de SAP y Openbravo siguiendo unas instrucciones de forma autónoma. Concretamente, se realizan procesos de empresa en las áreas de compras, ventas, producción y almacén de una compañía de fabricación de bicicletas configurada en el sistema a tal efecto, realizando

(cc)) BY-NC-ND 2017, Universitat Politècnica de València 
ciclos completos tal y como lo harían en una empresa real, desde que se crea un artículo nuevo, con sus componentes, pasando por su producción y almacenamiento, hasta la venta, facturación y cobro del mismo. Finalmente, después de realizar la práctica, los alumnos entregan, mediante una memoria, el resultado de los procesos que han realizado en la aplicación. De este modo, tanto al revisar la memoria, como mediante la observación durante la realización de las prácticas, el profesor puede evaluar la actividad realizada.

\subsection{Trabajo de campo}

El trabajo de campo consiste en visitar una pequeña o mediana empresa para analizar su sistema integrado de información para la gestión empresarial y proponer, de forma justificada, soluciones informáticas que permitan mejorar dicho sistema.

Cada grupo decide la empresa que quiere analizar, avisándolo previamente para no coincidir en la misma empresa diferentes grupos. Los grupos realizarán las gestiones necesarias para visitar la empresa, tomar imágenes de la misma y entrevistarse con las personas que consideran oportuno.

De esta manera, los alumnos ven el entorno real de una empresa y sus necesidades en cuanto a los sistemas de información y herramientas informáticas, y pueden hacer una comparativa con lo visto tanto en las clases de teoría, como en las prácticas de laboratorio, para ver cuáles son las necesidades y requerimientos reales que pueden ser cubiertos con las herramientas vistas en clase, y cuáles deberían desarrollarse a medida, dado que se trata de necesidades muy particulares que no están cubiertas en un sistema estándar, pero que son necesarias para el funcionamiento de la organización

Para estructurar el trabajo a desarrollar, a los alumnos se les proporciona una plantilla con los principales puntos que deberían abordar, este documento base incluye tres apartados principales:

i) Información relativa a la empresa (nombre, sector, misión, ubicación, empleados, facturación, ...)

ii) Descripción del Sistema de Información actual ( sistemas operacionales, sistemas para la toma de decisiones, sistemas de relación con el entorno, ...)

iii) Análisis de situación y propuestas de mejora (modificaciones/ampliaciones de las soluciones implantadas, cambios en la forma de utilización, propuesta de soluciones más adecuadas, ...)

\section{Evaluación}

Para la evaluación se ha utilizado la rúbrica diseñada en el marco del proyecto de competencias trasversales puesto en marcha por la UPV. En concreto se ha empleado la rúbrica CT13 de nivel de dominio III al tratarse de una asignatura de Master. En este nivel de dominio se busca: "Integrar correctamente las herramientas avanzadas del ámbito profesional". Para evaluar el grado alcance de la competencia, la rúbrica define tres indicadores: 1) Identificar las herramientas avanzadas y su utilidad, 2) Manejar las 
herramientas avanzadas y 3) Seleccionar y combinar las herramientas adecuadas para realizar un proyecto profesional o de investigación.

Resultado de aprendizaje: integrar correctamente las herramientas avanzadas del ámbito profesional.

\begin{tabular}{|c|c|c|c|c|}
\hline \multirow{2}{*}{ INDICADORES } & \multicolumn{4}{|c|}{ DESCRIPTORES } \\
\hline & D. No alcanzado & C. En desarrollo & B. Bien /adecuado & A. Excelente/ejemplar \\
\hline $\begin{array}{l}\text { Identifica las } \\
\text { herramientas } \\
\text { avanzadas y su } \\
\text { utilidad }\end{array}$ & $\begin{array}{l}\text { No identifica las } \\
\text { herramientas avanzadas }\end{array}$ & $\begin{array}{l}\text { Identifica las } \\
\text { herramientas avanzados } \\
\text { pero no reconoce su } \\
\text { utilidad }\end{array}$ & $\begin{array}{l}\text { Identifica las } \\
\text { herramientas avanzados } \\
\text { y su función principal }\end{array}$ & $\begin{array}{l}\text { Identifica funciones } \\
\text { adicionales de las } \\
\text { herramientas avanzados }\end{array}$ \\
\hline $\begin{array}{l}\text { Maneja las } \\
\text { herramientas } \\
\text { avanzadas }\end{array}$ & $\begin{array}{l}\text { No es capaz de manejar } \\
\text { las herramientas sin unas } \\
\text { instrucciones detalladas }\end{array}$ & $\begin{array}{l}\text { Maneja las herramientas } \\
\text { siguiendo unas } \\
\text { indicaciones detalladas }\end{array}$ & $\begin{array}{l}\text { Maneja las herramientas } \\
\text { de forma autónoma }\end{array}$ & $\begin{array}{l}\text { Maneja las herramientas } \\
\text { con soltura, explotando } \\
\text { todas sus funcionalidades }\end{array}$ \\
\hline $\begin{array}{l}\text { Selecciona y combina } \\
\text { las herramientas } \\
\text { adecuadas para } \\
\text { realizar un proyecto } \\
\text { profesional o de } \\
\text { investigación }\end{array}$ & $\begin{array}{l}\text { No identifica las } \\
\text { herramientas adecuadas } \\
\text { para el desarmollo del } \\
\text { proyecto }\end{array}$ & $\begin{array}{c}\text { Identifica las } \\
\text { herramientas a emplear } \\
\text { pero no las combina de } \\
\text { forma adecuada para el } \\
\text { desarrollo completo del } \\
\text { proyecto }\end{array}$ & $\begin{array}{c}\text { Combina } \\
\text { adecuadamente las } \\
\text { diferentes herramientas } \\
\text { para completar el } \\
\text { desarrollo del proyecto }\end{array}$ & $\begin{array}{l}\text { Vislumbra nuevas formas } \\
\text { de combinar } \\
\text { nerramientas para } \\
\text { completar el proyecto de } \\
\text { la forma más adecuada } \\
\text { posible, valorando sus } \\
\text { pros y contras }\end{array}$ \\
\hline
\end{tabular}

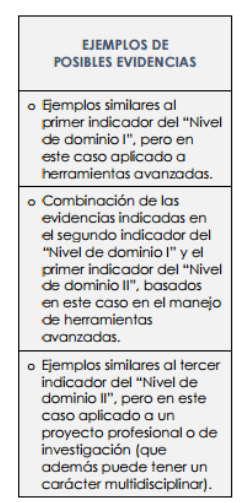

Fig. 1 Rubrica CT Instrumental Especifica

Como se puede observar en el cuadro de la derecha de la figura 1, la rúbrica propone también ejemplos de posibles evidencias para evaluar el nivel alcanzado en cada indicador.

Para la evaluación de los tres indicadores que incluye la rúbrica en el contexto de la asignatura se ha realizado con la recogida de las siguientes evidencias:

INDICADOR 1: Identifica las herramientas avanzadas y su utilidad

- Evidencia:

Cuestionario para identificar que el alumno realiza correctamente las prácticas, utiliza las herramientas adecuadas e identifica claramente sus funciones principales.

INDICADOR 2: Maneja las herramientas avanzadas

- Evidencia:

Memoria de ejecución de las prácticas para evidenciar que realiza la tarea siguiendo la secuencia de pasos correctamente y de forma autónoma.

INDICADOR 3: Selecciona y combina las herramientas adecuadas para prescribir una mejora en un contexto empresarial real

- Evidencias :

Informe y presentación del contexto empresarial y análisis del sistema de información.

Informe y presentación de propuestas de mejora del sistema de información de la empresa proponiendo una combinación de nuevas soluciones tecnológicas o modificaciones en el sistema actual.

(c)) BY-NC-ND 2017, Universitat Politècnica de València 


\section{Resultados y Conclusiones}

El avance en el desarrollo de las prácticas de laboratorio repercute claramente en un mejor manejo de las herramientas por parte de los alumnos, el trabajo de campo les aporta la verdadera posición que tienen las herramientas trabajadas en un contexto real, lo que les obliga a cuestionarse aspectos que no han sido previamente tratados en el aula y realizar propuestas en dicha realidad. Por último indicar que el uso de la rúbrica ha facilitado al profesorado el diseño de estas actividades.

\section{Referencias}

AKHTAR, J. (2013) Production planning and control with SAP ERP Boston: Galileo Pressa.

BOZA A.,CUENCA L. (2014) Strategy of online assessment in case study methodology. A particular case in the subject enterprise computers tools. ICERI2014 Proceedings, pp. 3207-3211.

BOZA A., CUENCA L., POLER R. \& MICHAELIDES Z.(2015): The interoperability force in the ERP field. Enterprise Information Systems Vol. 9 , Iss. 3,2015

CUENCA L., BOZA B., GORDO M.L., FERNANDEZ-DIEGO M., RUIZ L., ALARCON A., ALEMANY M.M.E. (2016) Extension of the 6-3-5 technique for incorporating creativity, innovation and entrepreneurship competence in higher education. ICERI2016 Proceedings, pp. 79-83.

GIL GÓMEZ, H, OLTRA BADENES, R., (2010) Evolution and trends of information systems for business management: the M-Business. A Review. DYNA vol.77 no.163

ICE - Instituto de Ciencias de la Educación. Universitat Politècncia de València. (2015) Proyecto Competencias Transversales UPV. <http://www.upv.es/entidades/ICE/info/U0724624.pdf $>$ [Consulta: 27 de marzo de 2017].

OLTRA BADENES, R. (2012). Sistemas Integrados de Gestión Empresarial: Evolución histórica y tendencias de futuro. Editorial Universitat Politècnica de València.

SAP University Alliances UPV (2017). http://suaupv.blogs.upv.es/. [Consulta: 27 de marzo de 2017].

UPV - UNIVERSITAT POLITÈCNICA DE VALÈNCIA - (2017). Competencias Transversales. http://www.upv.es/contenidos/COMPTRAN/info/955174normalc.html [Consulta: 10 de marzo de 2017]. 